\title{
ZERO INTEGRALS ON CIRCLES AND CHARACTERIZATIONS OF HARMONIC AND ANALYTIC FUNCTIONS
}

\author{
JOSIP GLOBEVNIK
}

\begin{abstract}
We determine the kernels of two circular Radon transforms of continuous functions on an annulus and use this to obtain a characterization of harmonic functions in the open unit disc which involves Poisson averages over circles computed at only one point of the disc and to obtain a version of Morera's theorem which involves only the circles which surround the origin.
\end{abstract}

\section{INTRODUCTION}

Suppose that $f$ is a continuous function on the open unit disc $\Delta$ in the complex plane. For each simple closed curve $\Gamma \subset \Delta$ bounding a domain $D$, $0 \in D$, let $F_{\Gamma}$ be the function which is continuous on $D \cup \Gamma$, harmonic in $D$ and which coincides with $f$ on $\Gamma$. It is known that if $F_{\Gamma}(0)=f(0)$ for each smooth curve $\Gamma$ bounding a strictly convex domain then $f$ is harmonic in $\Delta$. This is a special case of the main result of [5].

The starting point of the present investigation was the fact that the function $f$ is not necessarily harmonic if one assumes only that $F_{\Gamma}(0)=f(0)$ for each circle $\Gamma \subset \Delta$ surrounding the origin. In fact, for each $k \in N$ there is a function $f$ of class $\mathscr{C}^{k}$ on $\Delta$ such that $F_{\Gamma}(0)=f(0)$ for each circle $\Gamma \subset \Delta$ surrounding the origin and which is not harmonic in $\Delta$ ([5], see also $\S 9)$.

One of the results of the present paper is that if $F_{\Gamma}(0)=f(0)$ for each circle $\Gamma \subset \Delta$ which surrounds the origin then $f$ is harmonic in $\Delta$ under the additional assumption that it is infinitely differentiable at the origin, that is, if for each $n \in N$ there is a polynomial $P_{n}$ of degree $n$ such that

$$
f(z)=P_{n}(z, \bar{z})+Q_{n}(z) \quad(z \in \Delta)
$$

where $\lim _{z \rightarrow 0}|z|^{-n} Q_{n}(z)=0$. In fact, to get harmonicity it is enough to assume only that $F_{\Gamma}(0)=f(0)$ for each circle $\Gamma$ belonging to a family which is only slightly larger than the family of circles in $\Delta$ centered at the origin. Note that $f$ is infinitely differentiable at the origin if it is of class $\mathscr{C}^{\infty}$ in a neighbourhood of the origin, or more generally, if it belongs to $\mathscr{C}^{\infty}(\{0\})$, that is, if for each $k \in N$ there is a neighbourhood of the origin in which $f$ is of class $\mathscr{C}^{k}$.

Received by the editors February 15, 1988 and, in revised form, June 20, 1988.

1980 Mathematics Subject Classification (1985 Revision). Primary 30E20, 31A10; Secondary $53 C 65$. 
This characterization of harmonic functions is a consequence of our first main result which describes the Fourier coefficients of the continuous functions on an annulus $\Omega=\left\{\zeta \in C: R_{1}<|\zeta|<R_{2}\right\}$ which have zero average on each circle $\Gamma \subset \Omega$ that surrounds the origin. The second main result describes the Fourier coefficients of the continuous functions $f$ on $\Omega$ such that $\int_{\Gamma} f(z) d z=$ 0 for each circle that surrounds the origin. Its consequence is a version of Morera's theorem: If $f$ is continuous on $\Delta$ and infinitely differentiable at the origin and if $\int_{\Gamma} f(z) d z=0$ for each circle $\Gamma \subset \Delta$ which surrounds the origin then $f$ is analytic in $\Delta$. Again, one cannot drop the smoothness requirement at the origin since for each $k \in N$ there is a function $f$ of class $\mathscr{C}^{k}$ on $\Delta$ such that $\int_{\Gamma} f(z) d z=0$ for each circle $\Gamma \subset \Delta$ surrounding the origin and which is not analytic in $\Delta([3]$, see also $\S 9)$.

\section{The MAIN Results}

Let $0 \leq R_{1}<R_{2}$ and let $f$ be a continuous function on the annulus $\Omega=$ $\left\{\zeta \in C: R_{1}<|\zeta|<R_{2}\right\}$. For each $r, R_{1}<r<R_{2}$, and for each $n \in Z$, let

$$
f_{n}(r)=\frac{1}{2 \pi} \int_{0}^{2 \pi} e^{-i n \theta} f\left(r e^{i \theta}\right) d \theta .
$$

Thus, for each $r, R_{1}<r<R_{2}, \sum_{-\infty}^{\infty} f_{n}(r) e^{i n \theta}$ is the Fourier series of $f\left(r e^{i \theta}\right)$.

Let $\Gamma=\{\zeta \in C:|\zeta-z|=r\}$ and let $\varepsilon>0$. The $\varepsilon$-neighbourhood of the circle $\Gamma$ is the family of all circles of the form $\{\zeta \in C:|\zeta-w|=\rho$ with $|w-z|<\varepsilon$ and $|r-\rho|<\varepsilon, \rho>0$. Let $\mathscr{G}$ be a family of circles in $\Omega$ which surround the origin such that $\mathscr{G}$ contains a neighbourhood of each circle $\Gamma \subset \Omega$ centered at the origin. Note that $\mathscr{G}$ may be only slightly larger that the family of all circles in $\Omega$ centered at the origin.

If $\Gamma=\left\{a+e^{i \theta} b: 0 \leq \theta<2 \pi\right\}$ and if $\int_{0}^{2 \pi} f\left(a+e^{i \theta} b\right) d \theta=0$ then we will say that $f$ has zero average on $\Gamma$.

Theorem 1. Let $f$ be a continuous function on $\Omega$. The following are equivalent:

(i) $f$ has zero average on each circle $\Gamma \in \mathscr{G}$,

(ii) $f$ has zero average on each circle $\Gamma \subset \Omega$ which surrounds the origin,

(iii) $f_{0}(r)=0 \quad\left(R_{1}<r<R_{2}\right)$ and for each $n \in Z, n \neq 0$, there are numbers $a_{n 0}, a_{n 1}, \ldots, a_{n,|n|-1}$ such that

$$
f_{n}(r)=r^{-|n|}\left(a_{n 0}+a_{n 1} r^{2}+\cdots+a_{n,|n|-1} r^{2(|n|-1)}\right) \quad\left(R_{1}<r<R_{2}\right) .
$$

Theorem 2. Let $f$ be a continuous function on $\Omega$. The following are equivalent:

(i) $\int_{\Gamma} f(z) d z=0$ for each circle $\Gamma \in \mathscr{G}$,

(ii) $\int_{\Gamma} f(z) d z=0$ for each circle $\Gamma \subset \Omega$ which surrounds the origin,

(iii) $f_{-1}(r)=0 \quad\left(R_{1}<r<R_{2}\right)$ and for each $n \in Z, n \neq-1$, there are numbers $b_{n 0}, b_{n 1}, \ldots, b_{n,|n+1|-1}$ such that

$$
f_{n}(r)=r^{-|n|}\left(b_{n 0}+b_{n 1} r^{2}+\cdots+b_{n,|n+1|-1} r^{2(|n+1|-1)}\right) \quad\left(R_{1}<r<R_{2}\right) \text {. }
$$


Let $\mathscr{H}$ be a family of circles in $\Delta$ which surround the origin such that $\mathscr{H}$ contains a neighbourhood of each circle $\Gamma \subset \Delta$ centered at the origin.

Corollary 1. Let $f$ be a continuous function in $\Delta$ which is infinitely differentiable at the origin. If $F_{\Gamma}(0)=f(0)$ for each circle $\Gamma \in \mathscr{H}$ then $f$ is harmonic in $\Delta$. In particular, if $F_{\Gamma}(0)=f(0)$ for each circle $\Gamma \subset \Delta$ surrounding the origin then $f$ is harmonic in $\Delta$.

Corollary 2. Let $f$ be a continuous function in $\Delta$ which is infinitely differentiable at the origin. If $\int_{\Gamma} f(z) d z=0$ for each circle $\Gamma \in \mathscr{H}$ then $f$ is analytic in $\Delta$. In particular, if $\int_{\Gamma} f(z) d z=0$ for each circle $\Gamma \subset \Delta$ surrounding the origin then $f$ is analytic in $\Delta$.

An easy consequence of Theorems 1 and 2 is the following support theorem for the circular Radon transforms $f \mapsto \int_{\Gamma} f(z) d s$ and $f \mapsto \int_{\Gamma} f(z) d z$ which probably has a simple direct proof:

Corollary 3. Let $f$ be a continuous function on $\Omega$ such that for each $k \in N$ the function $z \mapsto\left(R_{2}-|z|\right)^{-k} f(z)$ is bounded as $|z| \rightarrow R_{2}$. If $f$ has zero average on each circle $\Gamma \in \mathscr{G}$ then $f$ vanishes identically on $\Omega$. If $\int_{\Gamma} f(z) d z=0$ for each circle $\Gamma \in \mathscr{G}$ then $f$ vanishes identically on $\Omega$.

The paper is organized as follows. We first prove that Corollaries 1-3 follow from Theorems 1 and $2(\S 3)$. Then we show that $\int_{\Gamma} f(z) d s=0$ or $\int_{\Gamma} f(z) d z=$ 0 for each circle $\Gamma \in \Omega$ surrounding the origin if and only if the same holds for each function $r e^{i \theta} \mapsto f_{n}(r) e^{i n \theta}(\S 4)$. This happens if and only if in each case $f_{n}$ satisfies a Volterra integral equation of the first kind whose kernel has a weak singularity on the diagonal $(\S 5)$. We look at the properties of these equations and iterate the kernels to get equations for the functions $r \mapsto f_{n}(r)\left(r_{0}-r\right)^{-1 / 2}$ with analytic kernels having zeros of order $|n|$ and $|n+1|$ on the diagonal $(\S 6)$. Since only the structure of bounded solutions of such equations has been studied in detail $[9,12]$ with only a remark being made in [9] about the general case we revisit [9] to show that the approximation procedure used there for bounded analytic solutions can be used also for unbounded smooth solutions $(\S \S 7,8)$. We then present examples of functions satisfying $\int_{\Gamma} f(z) d s=0$, $\int_{\Gamma} f(z) d z=0$ and show that using these examples one gets all solutions of the original integral equations and thus complete the proofs of Theorems 1 and 2 $(\S 9)$.

\section{PROOFS OF THE COROLLARIES}

Proposition 1. Let $D \subset C$ be an open disc, $0 \in D$, and let $\Gamma$ be its boundary. Assume that $F$ is a continuous function on $D \cup \Gamma$ which is harmonic in $D$. Then $F(0)=0$ if and only if the function $z \mapsto F(z) /|z|^{2}$ has zero average on $\Gamma$. 
Proof. Let $\Gamma=\left\{w+R e^{i \theta}: 0 \leq \theta<2 \pi\right\}, w=r e^{i \alpha}$. If $0 \leq \rho<1$ and $0 \leq \varphi<2 \pi$ then the Poisson formula gives

$$
F\left(w+\rho \operatorname{Re}^{i \varphi}\right)=\frac{1}{2 \pi} \int_{0}^{2 \pi} \frac{F\left(w+R e^{i t}\right)\left(1-\rho^{2}\right)}{1-2 \rho \cos (t-\varphi)+\rho^{2}} d t .
$$

Putting $\rho=r / R$ and $\varphi=\alpha+\pi$ we get

$$
\begin{aligned}
F(0) & =F\left(r e^{i \alpha}+(r / R) R e^{i(\alpha+\pi)}\right) \\
& =\frac{1}{2 \pi} \int_{0}^{2 \pi} \frac{F\left(r e^{i \alpha}+R e^{i t}\right)\left(1-\rho^{2}\right)}{1-2(r / R) \cos (t-\alpha-\pi)+r^{2} / R^{2}} d t \\
& =\left(R^{2}-r^{2}\right) \frac{1}{2 \pi} \int_{0}^{2 \pi} \frac{F\left(r e^{i \alpha}+R e^{i t}\right)}{\left|r e^{i \alpha}+R e^{i t}\right|^{2}} d t .
\end{aligned}
$$

This completes the proof.

Proof of Corollary 1, assuming Theorem 1. Suppose that $f$ is a continuous function on $\Delta$ which is infinitely differentiable at the origin and which satisfies $F_{\Gamma}(0)=f(0)$ for each circle $\Gamma \in \mathscr{H}$. It is enough to prove that $f$ is harmonic in $R \Delta$ for each $R<1$ so we may assume with no loss of generality that $f$ extends continuously to the closure $\bar{\Delta}$ of the unit disc.

Let $g$ be the function which is continuous on $\bar{\Delta}$, harmonic in $\Delta$ and which coincides with $f$ on the unit circle $b \Delta$ and put $h=f-g$. The function $h$ is continuous on $\bar{\Delta}$ and vanishes identically on $b \Delta$. Since $g$ is harmonic in $\Delta$ it follows that $h$ is infinitely differentiable at the origin and that $H_{\Gamma}(0)=0$ for each circle $\Gamma \in \mathscr{H}$. Proposition 1 now implies that the function $z \mapsto w(z)=$ $h(z) /|z|^{2}$ has zero average on each circle $\Gamma \in \mathscr{H}$. By Theorem 1 it follows that $w_{0}(r)=0 \quad(0<r<1)$ and that for each $n \in Z, n \neq 0$, there are numbers $a_{n i}, 0 \leq i \leq|n|-1$, such that

$$
w_{n}(r)=r^{-|n|}\left(a_{n 0}+a_{n 1} r^{2}+\cdots+a_{n,|n|-1} r^{2(|n|-1)}\right) \quad(0<r<1) .
$$

Since $h_{n}(r)=r^{2} w_{n}(r) \quad(0<r<1)$ it follows that

$$
r^{-|n|} h_{n}(r)=r^{-2|n|}\left(a_{n 0} r^{2}+a_{n 1} r^{4}+\cdots+a_{n,|n|-1} r^{2|n|}\right) \quad(0<r<1) .
$$

Fix $n \in Z, n \neq 0$. Since $h$ is infinitely differentiable at the origin there is a polynomial $P_{|n|}$ of degree $|n|$ such that $h(z)=P_{|n|}(z, \bar{z})+Q_{|n|}(z)$ where $\lim _{z \rightarrow 0}|z|^{-|n|} Q_{|n|}(z)=0$. This implies that there is a number $\alpha_{n}$ such that

$$
h_{n}(r)=\alpha_{n} r^{|n|}+\frac{1}{2 \pi} \int_{0}^{2 \pi} e^{-i n \theta} Q_{|n|}\left(r e^{i \theta}\right) d \theta
$$

and

$$
\lim _{r \rightarrow 0} r^{-|n|} h_{n}(r)=\alpha_{n} .
$$

Thus $a_{n j}=0 \quad(0 \leq j \leq|n|-2)$ and $h_{n}(r)=a_{n,|n|-1} r^{|n|} \quad(0<r \leq 1)$. Since $h$ vanishes identically on $b \Delta$ it follows that $h_{n}(1)=0$ which implies that 
$h_{n}(r)=0 \quad(0<r \leq 1)$. As this holds for each $n \in Z$ it follows that $h$ vanishes identically on $\Delta$, that is, $f$ coincides with $g$ on $\Delta$ which implies that $f$ is harmonic in $\Delta$. This completes the proof.

Proof of Corollary 2, assuming Theorem 2. Suppose that $f$ is a continuous function in $\Delta$ which is infinitely differentiable at the origin and which satisfies $\int_{\Gamma} f(z) d z=0$ for each circle $\Gamma \in \mathscr{H}$. By Theorem 2, $f_{-1}(r)=0 \quad(0<r<1)$ and for each $n \in Z, n \neq-1$, there are numbers $b_{n i}, 0 \leq i \leq|n+1|-1$, such that

$$
f_{n}(r)=r^{-|n|}\left(b_{n 0}+b_{n 1} r^{2}+\cdots+b_{n,|n+1|-1} r^{2(|n+1|-1)}\right) \quad(0<r<1) .
$$

As in the proof of Corollary 1 , since $f$ is infinitely differentiable at the origin it follows that for each $n \in Z, n \neq 0, \lim _{r \rightarrow 0} r^{-|n|} f_{n}(r)$, exists, that is,

$$
\lim _{r \rightarrow 0}\left(b_{n 0} r^{-2|n|}+b_{n 1} r^{-2|n|+2}+\cdots+b_{n,|n+1|-1} r^{-2|n|+2|n+1|-1}\right)
$$

exists. It follows that if $n<-1$ then $f_{n}(r)=0 \quad(0<r<1)$ and if $n \geq 0$ then $f_{n}(r)=b_{n,|n+1|-1} r^{n} \quad(0<r<1)$. Now, for each $r, 0<r<1, \sum_{-\infty}^{\infty} f_{n}(r) e^{i n \theta}$ is the Fourier series of $f\left(r e^{i \theta}\right)$ so on $[0,2 \pi]$ the function $f\left(r e^{i \theta}\right)$ is the uniform limit of its Cesàro means

$$
\begin{aligned}
\sigma_{m}\left(r e^{i \theta}\right) & =m^{-1}\left(f_{0}(r)+\sum_{k=-1}^{1} f_{k}(r) e^{i k \theta}+\cdots+\sum_{k=-(m-1)}^{m-1} f_{k}(r) e^{i k \theta}\right) \\
& =m^{-1}\left(b_{00}+\sum_{k=0}^{1} b_{k, k}\left(r e^{i \theta}\right)^{k}+\cdots+\sum_{k=0}^{m-1} b_{k, k}\left(r e^{i \theta}\right)^{k}\right) .
\end{aligned}
$$

The usual proof of the Fejér theorem [8] shows that the convergence is also uniform in $r, \rho_{1} \leq r \leq \rho_{2}$, for each $\rho_{1}, \rho_{2}, 0<\rho_{1}<\rho_{2}<1$, since $f$ is uniformly continuous in $\left\{\zeta: \rho_{1} \leq|\zeta| \leq \rho_{2}\right\}$ [3]. Since each $\sigma_{m}$ is analytic in $\Delta$ it follows that $f$ is analytic in $\Delta \backslash\{0\}$ and being continuous at $0, f$ is analytic in $\Delta$. This completes the proof.

Remark. The referee has kindly pointed out that if $f \in \mathscr{C}^{\infty}(\{0\})$ then the second part of Corollary 2 can be easily derived from the following consequence of an old result of A. M. Cormack: If $g \in \mathscr{C}^{\infty}(\Delta)$ has zero average on each circle $\Gamma \subset \Delta$ which passes through the origin then $g$ vanishes identically (for the proof see [1]). The reason is that once $f \in \mathscr{C}^{\infty}(\{0\})$ then it can be uniformly approximated by $\mathscr{C}^{\infty}$ functions with the same vanishing properties as $f$. Let $f \in \mathscr{C}^{\infty}(\Delta)$ and let $\int_{\Gamma} f(z) d z=0$ for each circle $\Gamma$ surrounding the origin. By continuity we have that $\int_{b D} f(z) d z=0$ for every disc $D \subset \Delta$, $0 \in b D$. By Green's formula, $\iint_{D} \partial f / \partial \bar{z} d z \wedge d \bar{z}=0$. It is easy to conclude that $\int_{b D} \partial f / \partial \bar{z} d s=0$ for all such discs $D$. It follows that $\partial f / \partial \bar{z}$ vanishes identically so $f$ is analytic on $\Delta$.

Proof of Corollary 3, assuming Theorems 1 and 2. The assumption implies that for each $n \in Z, k \in N$, the function $r \mapsto\left(R_{2}-r\right)^{-k} f_{n}(r)$ is bounded as 
$r \rightarrow R_{2}$. If $f$ has zero average on each circle $\Gamma \in \mathscr{G}$ then by Theorem 1 each $f_{n}$ extends to an analytic function in a neighbourhood of $\left(0, R_{2}\right]$ which is possible only if $f_{n}$ vanishes identically so $f$ vanishes identically in $\Omega$. In the same way, using Theorem 2, we see that if $\int_{\Gamma} f(z) d z=0$ for each $\Gamma \in \mathscr{G}$ then $f$ vanishes identically in $\Omega$. This completes the proof.

\section{ZERo INTEGRALS AND FoURIER COEFFICIENTS}

Let $\Gamma \subset C$ be a circle which surrounds the origin and whose center is not the origin. Let $A$ be the closed annulus obtained by rotating $\Gamma$ around the origin, that is,

$$
A=\bigcup_{0 \leq \alpha<2 \pi} e^{i \alpha} \Gamma .
$$

Let $R_{1}, R_{2}$ be such that $A=\left\{\zeta \in C: R_{1} \leq|\zeta| \leq R_{2}\right\}$. Let $f$ be a continuous function on $A$. For each $n \in Z$, let

$$
\Phi_{n}(z)=\frac{1}{2 \pi} \int_{0}^{2 \pi} e^{-i n \theta} f\left(e^{i \theta} z\right) d \theta \quad\left(R_{1} \leq|z| \leq R_{2}\right) .
$$

Note that if $z=r e^{i \alpha}$ then $\Phi_{n}(z)=f_{n}(r) e^{i n \alpha}$.

Lemma 1. The following are equivalent:

(i) $f$ has zero average on each circle $e^{i \alpha} \Gamma, 0 \leq \alpha<2 \pi$,

(ii) for each $n \in Z$ the function $\Phi_{n}$ has zero average on $\Gamma$.

Proof. Suppose that (i) holds. Let $\Gamma=\left\{a+e^{i \theta} b: 0 \leq \theta<2 \pi\right\}$. Let $n \in Z$. By the assumption, $\int_{0}^{2 \pi} f\left(e^{i \alpha}\left(a+e^{i \theta} b\right)\right) d \theta=0 \quad(0 \leq \alpha<2 \pi)$ so by the Fubini theorem

$$
\int_{0}^{2 \pi}\left[\frac{1}{2 \pi} \int_{0}^{2 \pi} e^{-i n \kappa} f\left(e^{i \alpha}\left(a+e^{i \theta} b\right)\right) d \alpha\right] d \theta=0
$$

which proves (ii). Conversely, assume that (ii) holds. Note first that (ii) implies that for each $n \in Z$ the function $\Phi_{n}$ has zero average on each circle $e^{i x} \Gamma$, $0 \leq \alpha<2 \pi$. For each $r, R_{1} \leq r \leq R_{2}, \sum_{-\infty}^{\infty} f_{n}(r) e^{i n \theta}=\sum_{-\infty}^{\infty} \Phi_{n}\left(r e^{i \theta}\right)$ is the Fourier series of $f\left(r e^{i \theta}\right)$. As in the proof of Corollary 2 we see that its Cesàro means

$$
\sigma_{m}\left(r e^{i \theta}\right)=m^{-1}\left(\Phi_{0}\left(r e^{i \theta}\right)+\sum_{k=-1}^{1} \Phi_{k}\left(r e^{i \theta}\right)+\cdots+\sum_{k=-(m-1)}^{m-1} \Phi_{k}\left(r e^{i \theta}\right)\right)
$$

converge to $f\left(r e^{i \theta}\right)$ uniformly on $A$ which implies (i). This completes the proof.

In almost the same way we prove the following lemma.

Lemma $\mathbf{1}^{\prime}$. The following are equivalent:

(i) $\int_{e^{i n} \Gamma} f(z) d z=0$ for each $\alpha, 0 \leq \alpha<2 \pi$,

(ii) $\int_{\Gamma} \Phi_{n}(z) d z=0$ for each $n \in Z$. 


\section{THE INTEGRAL EQUATIONS FOR THE FOURIER COEFFICIENTS}

For each $\rho, R, 0<\rho<R$, let $\Gamma_{\rho R}$ be the circle whose diameter is the segment $[-R, \rho]$ on the real axis. Further, for each $n \in N \cup\{0\}$ let $T_{n}$ be the Čebyšev polynomial of the first kind of degree $n$ [10]. We have $T_{n}(\cos \alpha)=$ $\cos n \alpha$ for each $\alpha$.

Lemma 2. Let $n \in Z$ and let $f_{n}$ be a continuous function on $[\rho, R]$. Let $\Phi_{n}\left(r e^{i \theta}\right)=f_{n}(r) e^{i n \theta} \quad(\rho \leq r \leq R, 0 \leq \theta<2 \pi)$ and let $s=\rho / R$. Write

$$
\begin{aligned}
& P_{n}(s, t)= T_{|n|}\left(\frac{s-t^{2}}{t(1-s)}\right)\left(t^{2}-s^{2}\right)^{-1 / 2}\left(1-t^{2}\right)^{-1 / 2} t \quad(s<t<1), \\
& Q_{n}(s, t)= {\left[2 t T_{|n+1|}\left(\frac{s-t^{2}}{t(1-s)}\right)+(1-s) T_{|n|}\left(\frac{s-t^{2}}{t(1-s)}\right)\right] } \\
& \times\left(t^{2}-s^{2}\right)^{-1 / 2}\left(1-t^{2}\right)^{-1 / 2} t \quad(s<t<1) .
\end{aligned}
$$

Then

(i) $\Phi_{n}$ has zero average on $\Gamma_{\rho R}$ if and only if

$$
\int_{s}^{1} f_{n}(R t) P_{n}(s, t) d t=0,
$$

(ii) $\int_{\Gamma_{\rho R}} \Phi_{n}(z) d z=0$ if and only if

$$
\int_{s}^{1} f_{n}(R t) Q_{n}(s, t) d t=0 .
$$

Proof. In polar coordinates $r$, the circle $\Gamma_{\rho R}$ is given by the equation

$$
\cos \varphi=\frac{R \rho-r^{2}}{r(R-\rho)}
$$

Differentiating this we get

$$
\frac{d \varphi}{d r} \sin \varphi=\frac{R \rho+r^{2}}{(R-\rho) r^{2}} \quad(\rho<r<R) .
$$

If $0<\varphi<\pi$ then (5.1) gives

$$
\sin \varphi=\left(1-\cos ^{2} \varphi\right)^{1 / 2}=\left(r^{2}-\rho^{2}\right)^{1 / 2}\left(R^{2}-r^{2}\right)^{1 / 2} r^{-1}(R-\rho)^{-1}
$$

so

$$
\frac{d \varphi}{d r}=\frac{R \rho+r^{2}}{r\left(r^{2}-\rho^{2}\right)^{1 / 2}\left(R^{2}-r^{2}\right)^{1 / 2}} \quad(\rho<r<R) .
$$

Similarly, if $-\pi<\varphi<0$,

$$
\frac{d \varphi}{d r}=-\frac{R \rho+r^{2}}{r\left(r^{2}-\rho^{2}\right)^{1 / 2}\left(R^{2}-r^{2}\right)^{1 / 2}} \quad(\rho<r<R) .
$$


In both cases we have

$$
\left(1+\left(r \frac{d \varphi}{d r}\right)^{2}\right)^{1 / 2}=r(R+\rho)\left(r^{2}-\rho^{2}\right)^{-1 / 2}\left(R^{2}-r^{2}\right)^{-1 / 2} \quad(\rho<r<R) .
$$

Now, writing

$$
\varphi(r)=\arccos \frac{R \rho-r^{2}}{r(R-\rho)} \quad(\rho<r<R)
$$

we have $\Gamma_{\rho R}=\left\{r e^{i \varphi(r)}: \rho \leq r \leq R\right\} \cup\left\{r e^{-i \varphi(r)}: \rho \leq r \leq R\right\}$ so if $\Psi$ is a continuous function on $\Gamma_{\rho R}$ its average on $\Gamma_{\rho R}$ is

$$
\pi^{-1} \int_{\rho}^{R}\left[\Psi\left(r e^{i \varphi(r)}\right)+\Psi\left(r e^{-i \varphi(r)}\right)\right]\left(r^{2}-\rho^{2}\right)^{-1 / 2}\left(R^{2}-r^{2}\right)^{-1 / 2} r d r .
$$

To prove (i), let $\Psi=\Phi_{n}$ and put $r=t R$. To prove (ii), observe that $\int_{\Gamma_{\rho R}} \Phi_{n}(z) d z=0$ if and only if the average of $\Psi(z)=\Phi_{n}(z)(z-(\rho-R) / 2)$ on $\Gamma_{\rho R}$ is zero and put $r=t R$. This completes the proof.

Lemma 3. Let $n \in Z$, let $0<R_{1}<R_{2}$ and assume that $f_{n}$ is a continuous function on $\left[R_{1}, R_{2}\right]$. Let $\Psi_{n}\left(r e^{i \theta}\right)=f_{n}(r) e^{i n \theta} \quad\left(R_{1} \leq r \leq R_{2}, 0 \leq \theta<2 \pi\right)$. Let $R_{1} / R_{2}<q<1$.

(i) If for each $\rho, R, R_{1} \leq \rho<R \leq R_{2}$, the function $\Psi_{n}$ has zero average on $\Gamma_{\rho R}$ then on $[q, 1]$ the function $t \mapsto f_{n}\left(t R_{2}\right)$ can be uniformly approximated by functions $\Phi$ of class $\mathscr{C}^{\infty}$ which satisfy

$$
\int_{s}^{1} \Phi(t) P_{n}(s, t) d t=0 \quad(q \leq s<1) .
$$

(ii) If for each $\rho, R, R_{1} \leq \rho<R \leq R_{2}, \int_{\Gamma_{\rho R}} \Psi_{n}(z) d z=0$, then on $[q, 1]$ the function $t \mapsto f_{n}\left(t R_{2}\right)$ can be uniformly approximated by functions $\Phi$ of class $\mathscr{C}^{\infty}$ which satisfy

$$
\int_{s}^{1} \Phi(t) Q_{n}(s, t) d t=0 \quad(q \leq s<1) .
$$

Proof. Suppose that for each $\rho, R, R_{1} \leq \rho<R \leq R_{2}, \Psi_{n}$ has zero average on $\Gamma_{\rho R}$. By Lemma 2

$$
\int_{s}^{1} f_{n}(t R) P_{n}(s, t) d t=0 \quad\left(R_{1} / R \leq s<1\right)
$$

holds for each $R, R_{1}<R \leq R_{2}$. In particular, if $R_{1}<R_{0}<R_{2}$ then for each $R, R_{0}<R<R_{2}$, the function $t \mapsto \Phi(t)=f_{n}(t R)$ satisfies

$$
\int_{s}^{1} \Phi(t) P_{n}(s, t) d t=0 \quad\left(R_{1} / R_{0}<s<1\right) .
$$

Choose $R_{0}<R_{2}$ so close to $R_{2}$ that $R_{1} / R_{0}<q$ and choose $\delta>0$ so small that $(1-\delta) R_{2}>R_{0}$. 
Let $\chi$ be a nonnegative $\mathscr{C}^{\infty}$ function on $R$ with support in $(1-\delta, 1)$ and such that $\int \chi(\omega) d \omega=1$. Since each function $t \mapsto f_{n}\left(\omega t R_{n}\right), 1-\delta<\omega<1$, satisfies (5.4) it follows by the Fubini theorem that also the function $t \mapsto \Phi(t)=$ $\int \chi(\omega) f_{n}\left(\omega t R_{2}\right) d \omega$ satisfies (5.4). The function $\Phi$ is of class $\mathscr{C}^{\infty}$ on $R \backslash\{0\}$ and if $\delta$ is chosen small enough then $\left|\Phi(t)-f_{n}\left(t R_{2}\right)\right|$ will be uniformly small on $\left[R_{1} / R_{0}, 1\right]$. This completes the proof of (i). We prove (ii) in the same way with $P_{n}$ replaced by $Q_{n}$.

\section{Properties of the integral equations}

To prove Theorems 1 and 2 we will need all smooth solutions of (5.2) and (5.3). We first mention two trivial special cases:

Proposition 2. Let $0<R_{1}<R_{2}$ and assume that $\Phi$ is a continuous function on $\left(R_{1}, R_{2}\right)$. If $\Psi\left(r e^{i \theta}\right)=\Phi(r) \quad\left(R_{1}<r<R_{2}, 0 \leq \theta<2 \pi\right)$ and if $\Psi$ has zero average on each circle $|\zeta|=r, R_{1}<r<R_{2}$, then $\Phi(r)=0 \quad\left(R_{1}<r<R_{2}\right)$. If $\Psi\left(r e^{i \theta}\right)=\Phi(r) e^{-i \theta} \quad\left(R_{1}<r<R_{2}, 0 \leq \theta<2 \pi\right)$ and if $\int_{|\zeta|=r} \Psi(z) d z=0$ for each $r, R_{1}<r<R_{2}$, then $\Phi(r)=0 \quad\left(R_{1}<r<R_{2}\right)$.

This shows that we will only have to consider (5.2) if $n \neq 0$ and (5.3) if $n \neq-1$.

Let $P_{n}$ and $Q_{n}$ be as in Lemma 3 .

Lemma 4. Let $0<\tau<1$ and let $\Phi$ be a continuous function on $[1-\tau, 1]$.

(i) If $n \in Z, n \neq 0$, and if $\Phi$ satisfies

$$
\int_{s}^{1} \Phi(t) P_{n}(s, t) d t=0 \quad(1-\tau<s<1),
$$

then

$$
\int_{0}^{p} K_{n}(p, t) g(t) d t=0 \quad(0<p<\tau)
$$

where $g(t)=h(1-t), h(t)=t^{-|n|+1}\left(1-t^{2}\right)^{-1 / 2} \Phi(t)$ and where $K_{n}$ is analytic in a neighbourhood of zero and is of the form

$$
K_{n}(p, t)=\sum_{i=0}^{|n|} b_{i} t^{|n|-i} p^{i}+\text { higher order terms in } t, p
$$

where $\sum_{i=0}^{|n|} b_{i} \neq 0$.

(ii) If $n \in Z, n \neq-1$, and if $\Phi$ satisfies

$$
\int_{s}^{1} \Phi(t) Q_{n}(s, t) d t=0 \quad(1-\tau<s<1),
$$

then

$$
\int_{0}^{p} L_{n}(p, t) g(t) d t=0 \quad(0<p<\tau)
$$


where $g(t)=h(1-t), h(t)=t^{-|n+1|+1}\left(1-t^{2}\right)^{-1 / 2} \Phi(t)$ and where $L_{n}$ is analytic in a neighbourhood of zero and is of the form

$$
L_{n}(p, t)=\sum_{i=0}^{|n+1|} c_{i} t^{|n+1|-i} p^{i}+\text { higher order terms in } t, p
$$

where $\sum_{i=0}^{|n+1|} c_{i} \neq 0$.

Proof. Let $n \in Z, n \neq 0$, and let $\Phi$ satisfy (6.1). Let $g$ be as in (i). Multiplying $(6.1)$ by $(1-s)^{n}$ we get

$$
\int_{s}^{1} L(s, t) h(t)(t-s)^{-1 / 2} d t=0 \quad(1-\tau<s<1)
$$

where

$$
L(s, t)=(t+s)^{-1 / 2} \sum_{i=0}^{|n|} p_{i}\left(s-t^{2}\right)^{|n|-i}(t(1-s))^{i}
$$

and where $T_{|n|}(x)=\sum_{i=0}^{|n|} p_{i} x^{|n|-i}$. Put $Q(s, t)=L(1-s, 1-t)$ and replace $t$ by $1-t$ and $s$ by $1-s$ in (6.3) to get

$$
\int_{0}^{s} Q(s, t) g(t)(t-s)^{-1 / 2} d t=0 \quad(0<s<\tau)
$$

where

$$
Q(s, t)=2^{-1 / 2} \sum_{i=0}^{|n|} p_{i}(2 t-s)^{|n|-i} s^{i}+\text { higher order terms in } s, t .
$$

Since $T_{|n|}(\cos x)=\cos n x$ we have $\sum_{i=0}^{|n|} p_{i}=1$ so $Q(s, s)=2^{-1 / 2} s^{|n|}+$ higher order terms in $s$. Thus we have shown that

$$
Q(s, t)=\sum_{i=0}^{|n|} a_{i} t^{|n|-i} s^{i}+\text { higher order terms in } s, t
$$

where $\sum_{i=0}^{|n|} a_{i} \neq 0$. One completes the proof of (i) by iterating the kernel as in $[9$, p. 155].

To prove (ii) let first $n \geq 0$. Let

$$
T_{n}(x)=\sum_{i=0}^{n} p_{i} x^{n-i}, \quad T_{n+1}(x)=\sum_{i=0}^{n+1} q_{i} x^{n+1-i} .
$$

Multiplying (6.2) by $(1-s)^{n+1}$ we get (6.3) where

$$
\begin{aligned}
L(s, t)=(t+s)^{-1 / 2}\left[2 t \sum_{i=0}^{n+1} q_{i}\left(s-t^{2}\right)^{i}(t(1-s))^{n+1-i}\right. & \\
& \left.+t(1-s)^{2} \sum_{i=0}^{n} p_{i}\left(s-t^{2}\right)^{i}(t(1-s))^{n-i}\right] .
\end{aligned}
$$


As before, this implies (6.4) where

$$
\begin{aligned}
Q(s, t)= & 2^{-1 / 2}\left[2 \sum_{i=0}^{n+1} q_{i}(2 t-s)^{i} s^{n+1-i}+\text { higher order terms in } s, t\right] \\
& +2^{-1 / 2} s^{2}\left[\sum_{i=0}^{n} p_{i}(2 t-s)^{i} s^{n-i}+\text { higher order terms in } s, t\right] \\
= & 2^{1 / 2} \sum_{i=0}^{n+1} q_{i}(2 t-s)^{i} s^{n+1-i}+\text { higher order terms in } s, t .
\end{aligned}
$$

Again, $Q(s, s)=2^{1 / 2} s^{n+1}+$ higher order terms in $s$, so

$$
Q(s, t)=\sum_{i=0}^{n+1} a_{i} t^{n+1-i} s^{i}+\text { higher order terms in } s, t
$$

where $\sum_{i=0}^{n+1} a_{i} \neq 0$. Together with [9, p. 155] this completes the proof of (ii) if $n \geq 0$. Now, let $n \leq-2$. Write $n=-m$. Since $T_{m}(x)=2 x T_{m-1}(x)-$ $T_{m-2}(x)[10]$ we have

$$
\begin{aligned}
& 2 t T_{m-1}\left(\frac{s-t^{2}}{t(1-s)}\right)+(1-s) T_{m}\left(\frac{s-t^{2}}{t(1-s)}\right) \\
& \quad=2 \frac{s}{t} T_{m-1}\left(\frac{s-t^{2}}{t(1-s)}\right)-(1-s) T_{m-2}\left(\frac{s-t^{2}}{t(1-s)}\right)
\end{aligned}
$$

so multiplying $(6.2)$ by $(1-s)^{m-1}$ we get $(6.3)$ where

$$
\begin{aligned}
L(s, t)=(t+s)^{-1 / 2}\left[2 \frac{s}{t} \sum_{i=0}^{m-1} p_{i}\left(s-t^{2}\right)^{m-1-i}(t(1-s))^{i}\right. & \\
& \left.-(1-s)^{2} t \sum_{i=0}^{m-2} q_{i}\left(s-t^{2}\right)^{m-2-i}(t(1-s))^{i}\right]
\end{aligned}
$$

and where $T_{m-1}(x)=\sum_{i=0}^{m-1} p_{i} x^{m-1-i}$ and $T_{m-2}(x)=\sum_{i=0}^{m-2} q_{i} x^{m-2-i}$. As before, (6.1) follows where

$$
Q(s, t)=2^{1 / 2} \sum_{i=0}^{m-1} p_{i}(2 t-s)^{m-1-i} s^{i}+\text { higher order terms in } s, t .
$$

Again, $Q(s, s)=2^{1 / 2} s^{m-1}+$ higher order terms in $s$, so

$$
Q(s, t)=\sum_{i=0}^{m-1} a_{i} t^{m-1-i} s^{i}+\text { higher order terms in } s, t
$$

where $\sum_{i=0}^{m-1} a_{i} \neq 0$. One completes the proof of (ii) as in [9, p. 155]. 


\section{THE INTEGRO-DIFFERENTIAL EQUATION}

Fix $n \in N$ and let $K$ be analytic in a neighbourhood of zero and of the form

$$
K(x, s)=\sum_{i=0}^{n} \beta_{i} s^{n-i} x^{i}+\text { higher order terms in } s, x
$$

where $\sum_{i=0}^{n} \beta_{i} \neq 0$. For the proofs of Theorems 1 and 2 we have to prove that if $\tau>0$ is small then the dimension of the space of all smooth solutions $g$ of the equation

$$
\int_{0}^{x} K(x, s) g(s) d s=0 \quad(0<x<\tau)
$$

such that $s^{1 / 2} g(s)$ is bounded on $(0, \tau)$, does not exceed $n$. For continuous functions $g$ on $[0, \tau)$ this follows from [12]. For bounded analytic functions $g$ this also follows from [9] where also a remark about the unbounded case was made. We follow [9] to show that the approximation procedure used there works also in our case.

Write $K(s, x)=\sum_{i=0}^{\infty} a_{i}(s)(x-s)^{i} / i$ ! where $a_{i}$ are analytic in a neighbourhood of zero and where the series converges in a neighbourhood of zero. By the properties of $K$,

$$
\left.\begin{array}{l}
a_{0} \text { has zero of order } n \text { at the origin and each } a_{i}, 1 \leq i \leq n-1, \\
\text { has zero of order at least } n-i \text { at the origin. }
\end{array}\right\}
$$

Suppose that $g$ is a smooth solution of (7.1) such that

$$
|g(s)| \leq C s^{-1 / 2} \quad(0<s<\tau)
$$

for some constant $C$. Since $g$ is smooth on $(0, \tau)$ and satisfies (7.1) and (7.3) one can differentiate (7.1) $n+1$ times to see that $g$ satisfies

$$
(D g)(x)=\int_{0}^{x} K_{n}(x, s) g(s) d s \quad(0<x<\tau)
$$

where $K_{n}$ is analytic in a neighbourhood of zero and where

$$
D g=\left(a_{0} g\right)^{(n)}+\left(a_{1} g\right)^{(n-1)}+\cdots+\left(a_{n} g\right) .
$$

By (7.2) zero is a singular point of $D$ which is of Fuchsian type, that is, it is a regular singular point of $D$.

Lemma 5. There are $k, 0 \leq k \leq n$, complex numbers $r_{i}, 1 \leq i \leq n$, $\operatorname{Re} r_{i}>0$ $(1 \leq i \leq k), \operatorname{Re} r_{i} \leq 0(k+1 \leq i \leq n)$, and functions $\Omega_{i}, H_{i}$, of the form

$$
\Omega_{i}(x)=\sum_{j=0}^{k_{i}} \Omega_{i j}(x)(\log x)^{j}, \quad H_{i}(x)=\sum_{j=0}^{k_{i}} H_{i j}(x)(\log x)^{j}
$$

where each $\Omega_{i j}$ and each $H_{i j}$ is analytic in a neighbourhood of zero such that for small $\tau>0$ the following holds: 
If $\Psi$ is a continuous function on $[0, \tau]$ such that $|\Psi(x)| \leq C x^{\eta} \quad(0<x<\tau)$ for some $\eta>0$ and for some constant $C$ then

$$
\begin{aligned}
y(x)= & \sum_{i=1}^{k} \Omega_{i}(x) x^{r_{i}} \int_{\tau / 2}^{x} t^{-r_{i}-1} H_{i}(t) \Psi(t) d t \\
& +\sum_{i=k+1}^{n} \Omega_{i}(x) x^{r_{i}} \int_{0}^{x} t^{-r_{i}-1} H_{i}(t) \Psi(t) d t
\end{aligned}
$$

satisfies $(D y)(x)=\Psi(x) \quad(0<x<\tau)$.

Proof. Each nonzero solution of $D y=0$ has the form

$$
y(x)=x^{r} \sum_{j=0}^{m} \Phi_{j}(x)(\log x)^{j}
$$

where each $\Phi_{j}$ is analytic in a neighbourhood of zero and at least one of the numbers $\Phi_{j}(0), 0 \leq j \leq m$, is different from zero [2]. Choose a fundamental system $y_{i}(x)=x^{r_{i}} \Omega_{i}(x), 1 \leq i \leq n$, where $\Omega_{i}$ are as in (7.5). The Wronskian has the form $W(x)=x^{r_{1}+\cdots+r_{n}-n(n-1) / 2} R(x)$ where $R$ is analytic in a neighbourhood of zero and satisfies $R(0) \neq 0$ [2, p. 77]. We complete the proof by using the variation of constants.

\section{SuCCESSIVE APPROXIMATIONS AND THE DIMENSION OF THE SPACE OF SOLUTIONS}

We keep the notation from $\S 7$. Define the operator

$$
\begin{aligned}
\left(L_{n} \varphi\right)(x)= & \sum_{i=1}^{k} \Omega_{i}(x) x^{r_{i}} \int_{\tau / 2}^{x} t^{-r_{i}-1} H_{i}(t)\left[\int_{0}^{t} K_{n}(t, s) \varphi(s) d s\right] d t \\
& +\sum_{i=k+1}^{n} \Omega_{i}(x) x^{r_{i}} \int_{0}^{x} t^{-r_{i}-1} H_{i}(t)\left[\int_{0}^{t} K_{n}(t, s) \varphi(s) d s\right] d t .
\end{aligned}
$$

If $\tau>0$ is small and if $\varphi$ is smooth on $(0, \tau)$ and such that $\varphi(x) x^{1 / 2}$ is bounded on $(0, \tau)$ then $L_{n} \varphi$ is well defined and smooth on $(0, \tau)$. Further, if $\tau$ is small then all the functions involved in the definition of $L_{n}$ are analytic in $\Sigma_{\tau}=\left\{r e^{i \alpha}: 0<r<\tau,|\alpha|<\pi / 4\right\}$ so if $\varphi$ is analytic in $\Sigma_{\tau}$ and such that $\varphi(x) x^{1 / 2}$ is bounded on $\Sigma_{\tau}$ then $L_{n} \varphi$ is well defined and analytic in $\Sigma_{\tau}$.

Using elementary estimates of the integrals we get

Lemma 6. There is a $\tau_{0}>0$ such that for each $\tau, 0<\tau<\tau_{0}$, and for each positive constant $C$ the following hold:

(i) if $\varphi$ is smooth on $(0, \tau)$ and if $|\varphi(x)| \leq C x^{-1 / 2}(0<x<\tau)$ then $L_{n} \varphi$ is bounded on $(0, \tau)$

(ii) if $\varphi$ is smooth on $(0, \tau)$ and if $|\varphi(x)| \leq C \quad(0<x<\tau)$ then $\left|\left(L_{n} \varphi\right)(x)\right|$ $\leq C / 2 \quad(0<x<\tau)$ 
(iii) if $\varphi$ is analytic in $\Sigma_{\tau}$ and if $|\varphi(x)| \leq C|x|^{-1 / 2} \quad\left(x \in \Sigma_{\tau}\right)$ then $L_{n} \varphi$ is bounded in $\Sigma_{\tau}$

(iv) if $\varphi$ is analytic in $\Sigma_{\tau}$ and if $|\varphi(x)| \leq C \quad\left(x \in \Sigma_{\tau}\right)$ then $\left|\left(L_{n} \varphi\right)(x)\right| \leq C / 2$ $\left(x \in \Sigma_{\tau}\right)$.

Now we use successive approximations:

Lemma 7. If $\tau>0$ is small enough then the following are equivalent

(i) $g$ is a smooth solution of (7.4) such that $g(s) s^{1 / 2}$ is bounded on $(0, \tau)$,

(ii) $g=g_{0}+L_{n} g_{0}+L_{n}^{2} g_{0}+\cdots$, where $g_{0}$ satisfies $D g_{0}=0$ on $(0, \tau)$ and

is such that $g_{0}(x) x^{1 / 2}$ is bounded on $\Sigma_{\tau}$ and where the series converges uniformly on $\Sigma_{\tau}$.

Proof. Let $\tau>0$ be so small that Lemma 6 holds and that in $2 \tau \Delta$ every solution of $D y=0$ has the form $x^{r} \sum_{j=0}^{m} P_{j}(x)(\log x)^{j}$ where $P_{j}, 0 \leq j \leq m$, are analytic in $2 \tau \Delta$. Suppose that (i) holds. By Lemma 6, $g_{0}=g-L_{n} g$ is smooth on $(0, \tau)$ and such that $g_{0}(s) s^{1 / 2}$ is bounded on $(0, \tau)$. By the definition of $L_{n}$ we have

$$
\begin{array}{rlr}
\left(D g_{0}\right)(x) & =(D g)(x)-\left(D\left(L_{n} g\right)\right)(x) \\
& =(D g)(x)-\int_{0}^{x} K_{n}(x, s) g(s) d s=0 \quad(0<x<\tau)
\end{array}
$$

since $g$ satisfies (7.4). Since $g_{0}(x) x^{1 / 2}$ is bounded on $(0, \tau)$ it follows that it is bounded in $\Sigma_{\tau}$. Since $g_{0}$ is analytic there Lemma 6 implies that the series (8.1) converges uniformly in $\Sigma_{\tau}$ and since $g_{0}=g-L_{n} g$ it follows by Lemma 6(ii) that the sum of the series (8.1) is $g$.

Conversely, suppose that (ii) holds. Write $L_{n}^{i} g_{0}=g_{i}$ so that $g=\sum_{i=0}^{\infty} g_{i}$. By the uniform convergence of the series in $\Sigma_{\tau}$ we have $D g=\sum_{i=0}^{\infty} D g_{i}$ on $(0, \tau)$ and

$$
\int_{0}^{x} K_{n}(x, s) g(s) d s=\sum_{i=0}^{\infty} \int_{0}^{x} K_{n}(x, s) g_{i}(s) d s \quad(0<x<\tau) .
$$

Since for each $i$,

$$
\left(D g_{i+1}\right)(x)=\int_{0}^{x} K_{n}(x, s) g_{i}(s) d s \quad(0<x<\tau)
$$

it follows that $g$ is a solution of (7.4). That $g(s) s^{1 / 2}$ is bounded on $(0, \tau)$ follows from Lemma 6 . This completes the proof.

Lemma 8. If $\tau$ is small enough then the dimension of the space of all smooth solutions $g$ of (7.4) such that $g(s) s^{1 / 2}$ is bounded on $(0, \tau)$ does not exceed $n$. Proof. Let $\tau>0$ be so small that Lemmas 6 and 7 hold. If $g_{0}$ is a solution of $D g_{0}=0$ such that $g_{0}(x) x^{1 / 2}$ is bounded in $\Sigma_{\tau}$ then by Lemma 6 the series (8.1) converges uniformly in $\Sigma_{\tau}$. Let $y_{1}, y_{2}, \ldots, y_{m}$ be the basis of the space 
of all solutions of $D y=0$ for which $y(x) x^{1 / 2}$ is bounded on $\Sigma_{\tau}$. For each $i$, $1 \leq i \leq m$, let $\Phi_{i}=y_{i}+L_{n} y_{i}+L_{n}^{2} y_{i}+\cdots$. By Lemma 7 a smooth function $g$ on $(0, \tau)$ such that $g(s) s^{1 / 2}$ is bounded on $(0, \tau)$ satisfies (7.4) if and only if it is a linear combination of the functions $\Phi_{i}, 1 \leq i \leq m$. This completes the proof.

Now, using Lemma 4 and $\S 7$ we get the following consequence:

Lemma 9. Let $n \in N, n \neq 0$. There is some $q_{0}<1$ such that for each $q$, $q_{0}<q<1$, the dimension of the space of smooth functions $\Phi$ on $[q, 1]$ which satisfy (5.2) does not exceed $|n|$.

Let $n \in N, n \neq-1$. There is some $q_{0}<1$ such that for each $q, q_{0}<q<1$, the dimension of the space of smooth functions $\Phi$ on $[q, 1]$ which satisfy $(5.3)$ does not exceed $|n+1|$.

\section{EXAMPLES AND THE PROOFS OF THEOREMS 1 AND 2}

Proposition 3. Let $n \in N$. Each of the functions $z \mapsto z^{n-k} \bar{z}^{-k}, 1 \leq k \leq n$, has zero average on each circle that surrounds the origin.

Proof [5]. Fix $k, 1 \leq k \leq n$, and let $\varphi(z)=z^{n-k} \bar{z}^{-k}$. Let $0 \leq|a|<|b|$. Then

$$
\begin{aligned}
\int_{0}^{2 \pi} \varphi\left(a+e^{i \theta} b\right) d \theta & =\int_{0}^{2 \pi}\left(a+e^{i \theta} b\right)^{n-k}\left(\bar{a}+e^{-i \theta} \bar{b}\right)^{-k} d \theta \\
& =-i \int_{0}^{2 \pi}\left(\bar{a} e^{i \theta}+\bar{b}\right)^{-k}\left(e^{i \theta}\right)^{k-1}\left(a+e^{i \theta} b\right)^{n-k} i e^{i \theta} d \theta \\
& =-i \int_{b \Delta}(\bar{a} w+\bar{b})^{-k} w^{k-1}(a+b w)^{n-k} d w .
\end{aligned}
$$

Since $|b|>|a|$, since $k \geq 1$ and since $n-k \geq 0$ the integrand in the last integral is analytic in a neighbourhood of $\bar{\Delta}$ so the last integral is zero. This completes the proof.

Example [5]. If $n \in N$ then the function

$$
\varphi(z)=\left\{\begin{array}{l}
\bar{z}^{-1} z^{n+2} \quad(z \neq 0), \\
0 \quad(z=0)
\end{array}\right.
$$

is of class $\mathscr{C}^{n}$ on $C$. By Propositions 1 and 3 we have $\Phi_{\Gamma}(0)=\varphi(0)$ for every circle $\Gamma$ that surrounds the origin, yet $\varphi$ is not harmonic in $\Delta$.

Proposition 4. Let $0<a<b \leq 1$ and let $n \in N$. The uniform limit on $(a, b)$ of a sequence of polynomials of the form $a_{0}+a_{1} x^{2}+\cdots+a_{n} x^{2 n}$ is a polynomial of the same form.

The proof is easy and we omit it.

Proof of Theorem 1. It is enough to prove the equivalence of (ii) and (iii). Let $f$ be a continuous function on $\Omega$. Assume that $f$ satisfies (iii). By Proposition 
3 for each $n \in Z$ the function $r e^{i \theta} \mapsto f_{n}(r) e^{i n \theta}$ has zero average on each circle $\Gamma \subset \Omega$ surrounding the origin so by Lemma $1 f$ satisfies (ii).

Suppose that $f$ satisfies (ii). By Proposition 2, $f_{0}(r)=0 \quad\left(R_{1}<r<R_{2}\right)$. Let $n \in Z, n \neq 0$. By Proposition 3 and Lemma 2 each of the functions $\Phi_{k}(t)=t^{|n|-2 k}, 1 \leq k \leq n$, satisfies (5.2). By Lemma 9 it follows that if $q_{0}<q<1$ then each smooth solution of (5.2) is a linear combination of the functions $\Phi_{k}, 1 \leq k \leq n$. By Proposition 4 the uniform limit on $[q, 1]$ of a sequence of linear combinations of $\Phi_{k}, 1 \leq k \leq n$, is again a linear combination of $\Phi_{k}, 1 \leq k \leq n$. If $R_{1}<R<R_{2}$ and if $q>R_{1} / R$ then it follows by Lemma 3 that on $[q, 1]$ the function $t \mapsto f_{n}(t R)$ is a linear combination of $\Phi_{k}, 1 \leq k \leq n$. As this holds for every $R, R_{1}<R<R_{2}$, the proof is complete.

Proposition 5. If $n \in Z, n \geq 0$, then each of the functions $F_{k}(z)=z^{n-k} \bar{z}^{-k}$, $0 \leq k \leq n$, satisfies $\int_{\Gamma} F_{k}(z) d z=0$ for each circle $\Gamma$ surrounding the origin. If $n \in Z, n \leq-2$ then each of the functions $G_{k}(z)=\bar{z}^{k} z^{n+k}, 0 \leq k \leq-n-2$, satisfies $\int_{\Gamma} G_{k}(z) d z=0$ for each circle $\Gamma$ surrounding the origin.

Proof. Let $n \geq 0$ and let $0 \leq k \leq n$. Let $0<|a|<|b|$ and let $\Gamma=\{a+$ $\left.e^{i \theta} b: 0 \leq \theta<2 \pi\right\}$. If $F_{k}(z)=z^{n-k} \bar{z}^{-k}$ then

$$
\begin{aligned}
\int_{\Gamma} F_{k}(z) d z & =\int_{0}^{2 \pi}\left(a+e^{i \theta} b\right)^{n-k}\left(\bar{a}+e^{-i \theta} \bar{b}\right)^{-k} i b e^{i \theta} d \theta \\
& =b \int_{0}^{2 \pi} e^{i k \theta}\left(a+e^{i \theta} b\right)^{n-k}\left(\bar{a} e^{i \theta}+\bar{b}\right)^{-k} i e^{i \theta} d \theta \\
& =b \int_{b \Delta} w^{k}(a+w b)^{n-k}(\bar{a} w+\bar{b})^{-k} d w=0
\end{aligned}
$$

since the integrand in the last integral is analytic in a neighbourhood of $\bar{\Delta}$. Let $n \leq-2$. Write $n=-m$ and let $G_{k}(z)=\bar{z}^{k} z^{-m+k}, 0 \leq k \leq m-2$. We have

$$
\begin{aligned}
\overline{\int_{\Gamma} G_{k}(z) d z} & =\overline{\int_{0}^{2 \pi}\left(\bar{a}+e^{-i \theta} \bar{b}\right)^{k}\left(a+e^{i \theta} b\right)^{-m+k} i b e^{i \theta} d \theta} \\
& =-\int_{0}^{2 \pi}\left(a+e^{i \theta} b\right)^{k}\left(\bar{a}+e^{-i \theta} \bar{b}\right)^{-m+k} i \bar{b} e^{-i \theta} d \theta \\
& =-\int_{0}^{2 \pi}\left(a+e^{i \theta} b\right)^{k} e^{i(m-k) \theta}\left(\bar{a} e^{i \theta}+\bar{b}\right)^{-m+k} i \bar{b} e^{-i \theta} d \theta \\
& =-\bar{b} \int_{b \Delta}(a+w b)^{k} w^{m-k-2}(\bar{a} w+\bar{b})^{-m+k} d w=0
\end{aligned}
$$

since the integrand in the last integral is analytic in a neighbourhood of $\bar{\Delta}$.

Example [3]. If $n \in N$ then the function

$$
\varphi(z)=\left\{\begin{array}{l}
\bar{z}^{-1} z^{n+2} \quad(z \neq 0), \\
0 \quad(z=0)
\end{array}\right.
$$


is of class $\mathscr{C}^{n}$ on $C$. By Proposition 5 we have $\int_{\Gamma} \varphi(z) d z=0$ for every circle $\Gamma$ that surrounds the origin, yet $\varphi$ is not analytic in $\Delta$.

Proof of Theorem 2. It is enough to prove the equivalence of (ii) and (iii). Let $f$ be a continuous function on $\Omega$. If $f$ satisfies (iii) then by Proposition 5 for each $n \in Z$ the function $r e^{i \theta} \mapsto \Psi_{n}\left(r e^{i \theta}\right)=f_{n}(r) e^{i n \theta}$ satisfies $\int_{\Gamma} \Psi_{n}(z) d z=0$ for each circle $\Gamma \subset \Omega$ surrounding the origin so by Lemma $1^{\prime} f$ satisfies (ii).

Suppose that $f$ satisfies (ii). By Proposition 2, $f_{-1}(r)=0 \quad\left(R_{1}<r<R_{2}\right)$. Let $n \in Z, n \neq-1$. If $n \geq 0$ then by Proposition 5 and Lemma 2 each of the functions $\Phi_{k}(t)=t^{-|n|+2 k}, 0 \leq k \leq n$, satisfies (5.3). As in the proof of Theorem 1 it follows that if $R_{1}<R<R_{2}$ and if $q>R_{1} / R$ then on $[q, 1]$ the function $t \mapsto f_{n}(t R)$ is a linear combination of $\Phi_{k}, 0 \leq k \leq n$. As this holds for every $R, R_{1}<R<R_{2}$, (iii) follows for $n \geq 0$. If $n \leq-2$ then by Proposition 5 and Lemma 2 each of the functions $\Phi_{k}(t)=t^{-|n|+2 k}$, $0 \leq k \leq|n|-2$, satisfies (5.3) and again (iii) follows for $n \leq-2$. This completes the proof.

Acknowledgements. The author is indebted to Professor Ivan Vidav for helpful and stimulating discussions. In particular, when the author told him that he proved Theorem 1 for functions belonging to $\mathscr{C}^{\infty}(\{0\})$ Professor Vidav proposed the appropriate smoothness assumption at the origin. A major part of this work was done during the month of November 1987 which the author spent at the Research Institute for Mathematical Sciences of Bar-Ilan University, Ramat Gan, Israel. He wishes to thank Professor Lawrence Zalcman for the invitation and for several very interesting discussions.

This work was supported in part by the Republic of Slovenia Science Foundation.

\section{REFERENCES}

1. A. M. Cormack and E. T. Quinto, A Radon transform on spheres through the origin in $R^{n}$ and applications to the classical Darboux equation, Trans. Amer. Math. Soc. 260 (1980), 575-581.

2. A. R. Forsyth, Theory of differential equations, Vol. 4, Dover, New York, 1959.

3. J. Globevnik, Analyticity on rotation invariant families of curves, Trans. Amer. Math. Soc. 280 (1983), 247-254.

4. __ Testing analyticity on rotation invariant families of curves, Trans. Amer. Math. Soc. 306 (1988), 401-410.

5. J. Globevnik and W. Rudin, A characterization of harmonic functions, Indag. Math. 91 (1988), 419-426.

6. E. Goursat, Cours d'analy'se mathématique, Vol. II, Gauthier-Villars, Paris, 1933.

7. S. Helgason, The Radon transform, Progress in Math., No. 5, Birkhäuser, Boston, Mass., Basel and Stuttgart, 1980.

8. K. Hoffman, Banach spaces of analytic functions, Prentice-Hall, Englewood Cliffs, N. J., 1962.

9. T. Lalesco, Sur l'equation de Volterra, J. Math. (6) 4 (1908), 125-202.

10. W. Magnus, F. Oberhettinger and R. P. Soni, Formulas and theorems for the special functions of mathematical physics, Grundlagen. Math. Wiss., Bd. 52, Springer, Berlin, 1966. 
11. V. G. Romanov, Integral geometry and inverse problems for hyperbolic equations, Tracts in Natural Philos., Vol. 26, Springer, Berlin, 1974.

12. V. Volterra and J. Pérès, Théorie générale des fonctionelles, Gauthier-Villars, Paris, 1936.

Institute of Mathematics, Physics and Mechanics, E. K. University of Ljubljana, LuUbluana, Yugoslavia

Current address: Department of Mathematics, University of Washington, Seattle, Washington 98195 